\title{
Secondary erythromelalgia successfully treated with patient-controlled epidural analgesia and interferon $\alpha-2 b$ : A case report and review of the literature
}

\author{
XINGZHI LI ${ }^{1}$, YAOMIN $\mathrm{LI}^{2}$, YAO QU ${ }^{1}$ and LAIJIN LU ${ }^{3}$ \\ ${ }^{1}$ Department of Pain Management, First Hospital of Jilin University, Changchun, Jilin 130021; \\ ${ }^{2}$ Department of Rehabilitation, Fushun Central Hospital, Fushun, Liaoning 113000; \\ ${ }^{3}$ Department of Hand Surgery, First Hospital of Jilin University, Changchun, Jilin 130021, P.R. China
}

Received November 18, 2014; Accepted November 30, 2015

DOI: 10.3892/etm.2016.3088

\begin{abstract}
Erythromelalgia (EM) is a debilitating neurovascular disease that is refractory to numerous treatment modalities. The present study reported the case of a 72-year-old woman diagnosed with EM secondary to polycythemia vera (PV), who was effectively treated with the use of patient-controlled epidural analgesia (PCEA) and interferon $\alpha-2 b$ therapy. The study aimed to provide clinical information on this condition based on the findings of the present case and previously-reported cases. PCEA containing local anesthetics and dexamethasone or fentanyl resulted in nearly complete relief of symptoms. Furthermore, continuous use of interferon $\alpha-2 b$ for the treatment of the PV completely relieved the EM symptoms. In conclusion, PCEA and interferon $\alpha-2 b$ may be promising treatments for EM secondary to $\mathrm{PV}$.
\end{abstract}

\section{Introduction}

Erythromelalgia (EM) is a rare neurovascular syndrome characterized by intermittent or continuous erythema, a burning sensation and an elevated skin temperature in the hands, feet and, less commonly, in the nose and ears (1). In a previous study, the incidence of EM was shown to be 0.36 per 100,000 people per year (2). This debilitating disease can greatly affect the patients' quality of life. Although several therapeutic options exist, including conservative treatments

Correspondence to: $\mathrm{Dr}$ Yao Qu, Department of Pain Management, First Hospital of Jilin University, 71 Xinmin Street, Changchun, Jilin 130021, P.R. China

E-mail: quyao.2002@aliyun.com

Professor Laijin Lu, Department of Hand Surgery, First Hospital of Jilin University, 71 Xinmin Street, Changchun, Jilin 130021, P.R. China

Email: lulaijin@hotmail.com

Key words: erythromelalgia, patient-controlled epidural analgesia, interferon, polycythemia vera such as aspirin, serotonin reuptake inhibitors, tricyclic antidepressants and anticonvulsants, and invasive approaches including performing a sympathetic block or sympathectomy, no method is consistently effective in managing the various symptoms (3). Previous investigations of EM reported prolonged remission of symptoms with epidural infusions, intrathecal infusion and sympathetic ganglion block with local anesthetics (4-6). Polycythemia vera (PV) is a myeloproliferative disease, which is occasionally accompanied by cutaneous manifestations, including EM (7). The present study reports the case of a 72-year-old woman with EM secondary to PV who responded well to patient-controlled epidural analgesia (PCEA) and interferon $\alpha-2 b$ therapy.

\section{Case report}

A 72-year-old woman presented at the Department of Pain Management at the First Hospital of Jilin University (Changchun, China) on 18th August 2011 with a 1-year history of continuous, severe pricking pain with redness and swelling of the bilateral forefeet and toes. The patient experienced high temperature in the feet and avoided wearing socks and shoes. Standing exacerbated the symptoms, which were relieved by elevation or exposure of the lower extremities to cold. The face and bilateral hands of the patient were ruddy and asymptomatic. The patient's pertinent medical history included hypertension, heart disease and cerebral infarction with secondary anomic aphasia 4 months prior to admission to our hospital, while her family history was noncontributory. The patient began taking aspirin (100 mg/day) regularly following the cerebral infarction for secondary prevention of cerebrovascular events with no improvement of the EM symptoms.

Physical examination revealed moderate bilateral erythema, edema and an increased skin temperature of the soles of the feet and toes (Fig. 1A). Hyperalgesia and allodynia were present in the affected area, with the toes being more severely affected. The visual analog scale (VAS) score of the patient was 8 (8). Vascular Doppler investigation of the right lower extremity and electrocardiography examination revealed normal findings. A complete blood count (CBC) 
A

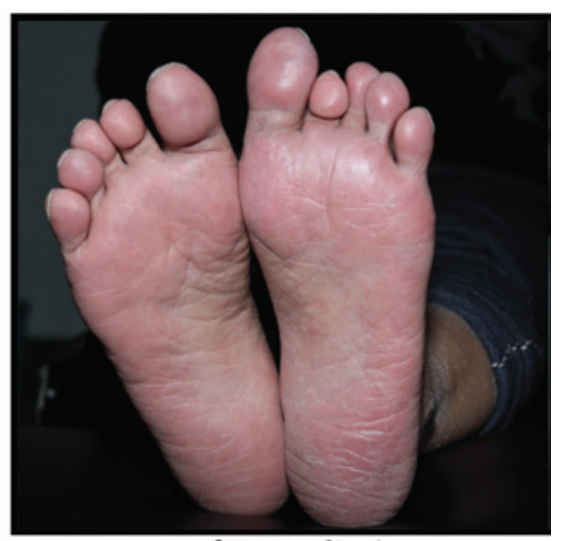

Before PCEA

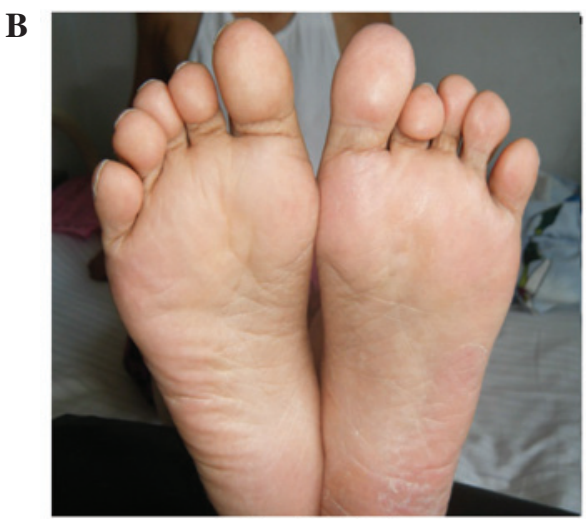

After PCEA

Figure 1. (A) Moderate bilateral erythema and edema of the soles and toes prior to PCEA. (B) The bilateral erythema and edema of the affected regions resolved following PCEA. PCEA, patient-controlled epidural analgesia.

revealed elevated red blood cell (RBC) count, hemoglobin (HGB) level and platelet (PLT) count (Table I).

A diagnosis of secondary EM was established based on the patient's clinical manifestations, laboratory data and lack of family history of this disease. On day 2 following admission, a lumbar epidural catheter was inserted $4.5 \mathrm{~cm}$ cephalad at the L3-4 interspace. The patient was placed on PCEA with $0.2 \%$ ropivacaine, $0.2 \%$ lidocaine and dexamethasone at $100 \mu \mathrm{g} / \mathrm{ml}$ after a test dose. The background infusion rate was $2 \mathrm{ml} / \mathrm{h}$ with self-administered 5 - $\mathrm{ml}$ boluses at intervals of $\leq 15 \mathrm{~min}$. The patient reported remarkable pain relief within $10 \mathrm{~h}$. In addition, the abnormal physical signs were nearly resolved. The PCEA was continued for 1 week with no complications. After 1 week of treatment, the erythema, edema and elevated skin temperature were completely resolved (Fig. 1B). Furthermore, the hyperalgesia and allodynia had nearly disappeared, allowing the patient to walk unassisted while wearing socks and shoes. After 3 days from the discontinuation of PCEA, the patient was discharged from the hospital on 29th August 2011 with a VAS score of 1 .

The patient returned on 14th February 2012 with a 10-day history of the same symptoms and signs. A VAS score of 8 was reported, and certain CBC parameters were found to be increased (Table I). PCEA ( $0.2 \%$ ropivacaine, $0.1 \%$ lidocaine and $5 \mu \mathrm{g} / \mathrm{ml}$ fentanyl) was initiated and continued for 13 days. Once again, the patient exhibited remarkable

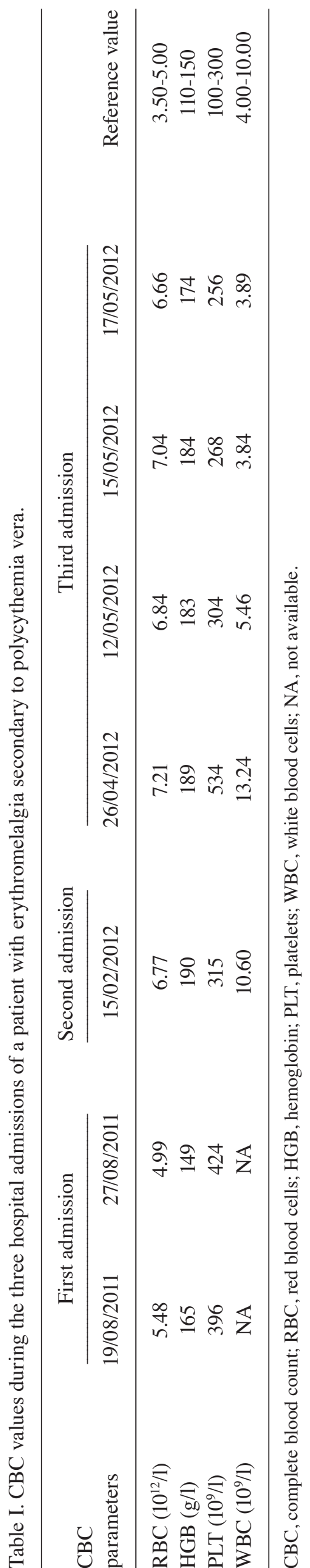


improvement, with a VAS score of 1 at the time of discharge on 28th February 2012. On 25th April 2012, the patient visited the Department of Hematology at the First Hospital of Jilin University, due to repeated abnormal CBC parameters with a constellation of less severe EM symptoms (VAS score, 5). All the previously measured $\mathrm{CBC}$ parameters were again found to be increased (Table I). A bone marrow biopsy indicated myeloproliferative disease, and an abdominal ultrasound revealed mild splenomegaly. Genetic testing revealed positivity for JAK2 V617F and negativity for JAK2 exon 12. The patient was diagnosed with PV according to criteria outlined by the World Health Organization (WHO) (9), which included an elevated level of HGB, the presence of the JAK2V617F mutation, and the detection of myeloproliferative disease. The patient was placed on recombinant human interferon $\alpha-2 b$ therapy to treat the PV, which resulted in complete relief of the EM symptoms. After 2 weeks, the PLT count had returned to the normal levels, with slight decreases in the RBC count and HGB level (Table I). The patient was then discharged on 17th May 2012 with complete remission of the symptoms and signs. At the 16-month follow-up, the patient remained asymptomatic with continuous recombinant interferon treatment. At the 43-month follow-up, the patient had experienced several episodes of recurrence following discontinuation of the interferon treatment due to side effects, including anorexia and precordial distress. Currently, the patient is not under treatment and suffers from mild symptoms of EM. Written informed consent was obtained from the patient.

\section{Discussion}

EM is a rare syndrome that has not been as widely studied as other functional vascular conditions, including Raynaud's phenomenon and acrocyanosis (10). This syndrome is divided into the primary EM (PEM) and secondary EM (SEM) types. Gain-of-function mutations in SCN9A, which encodes the $\alpha$-subunit of the voltage-gated sodium channel Nav1.7, have been found to be the main cause of PEM (11). By contrast, SEM has been described in association with various disorders, with essential thrombocythemia (ET) and PV as the first and second most common causes, respectively (3). In the patient of the present study, repeated CBC abnormalities suggested the presence of thrombocythemia and erythrocythemia, indicating a possible diagnosis of ET and/or PV. It was necessary to determine which condition contributed to EM in the present case. However, establishing the correct diagnosis was challenging, since thrombocythemia can occur as a result of both ET and PV, and serves as the fundamental cause of EM (7). Due to limited knowledge of this condition and a lack of equipment in the Department of Pain Management at our hospital, the diagnosis of PV was confirmed by a hematologist from the Department of Hematology, according to the criteria outlined by the WHO (9). The most appropriate diagnosis in the present case was PV associated with thrombocythemia (PVAT). Thrombocythemia-induced EM can be considered as a microvascular ischemic complication of PV. In patients with PVAT, a high hematocrit and increased whole blood viscosity aggravate the PLT-mediated microvascular syndrome, resulting in major arterial complications (7); this may explain the complicated medical history of the present patient. In the current case, interferon treatment inhibited the proliferation of cells, particularly PLTs, more than it affected the RBC and HGB levels, further illustrating that PLTs play a vital role in PVAT. Low-dose aspirin (100 mg/day) resulted in no improvement, indicating that aspirin may not be effective in patients with this type of SEM. Interferon therapy, which targets the underlying disease, should be considered as an alternative. In a recent study, interferon $\alpha$ was associated with a significantly lower incidence of EM than was hydroxyurea without severe hematological adverse events in patients with JAK2 V617F-positive PV (12).

According to the shunting hypothesis, EM symptoms are caused by tissue hypoxia, which is induced by a maldistribution of skin microvascular blood flow with increased thermoregulatory flow through arteriovenous shunts and an inadequate nutritive perfusion to normal skin capillaries $(13,14)$. If available blood is shunted away from normal skin capillaries, the skin will be hypoxic. The shunting hypothesis is generally considered to be the final common pathway for PEM and SEM from a pathophysiological perspective; however, histopathological investigations reported that the alterations associated with PEM were different from those associated with SEM (15-17). In a prospective study, Kalgaard et al (15) reported histopathological findings characterized by capillary proliferation or vascular damage in 31 of 49 specimens, mainly from patients with PEM. These nonspecific alterations indicated the presence of skin hypoxia secondary to increased arteriovenous shunting and insufficient capillary flow, which is compatible with the shunting hypothesis supported by Mørk et al $(13,14)$.

The use of an epidural infusion with local anesthetics and dexamethasone or fentanyl provided pronounced symptom relief in the present patient. A possible explanation may be sympathetic denervation, which improved the blood flow to the skin at the capillary level, thus reversing the chronic ischemic status secondary to increased arteriovenous shunting triggered by hypersensitive PLT-mediated arteriolar thrombosis. This explanation is supported by successful outcomes of other similarly aggressive techniques, including intrathecal infusion and sympathetic ganglion block, the therapeutic effects of which are mediated through inhibition of the sympathetic nerves $(5,6)$. Further histological and physiological studies of autonomic nerve function in patients with SEM are required to illustrate whether SEM is sympathetically mediated.

In conclusion, the present study reported a typical case of EM secondary to PV. The patient responded well to PCEA with infusion of two local anesthetics plus dexamethasone or fentanyl. No unacceptable side effects developed during the treatment. Interferon therapy, which inhibits PLT generation, was more effective than aspirin, which inhibits PLT aggregation. PCEA acts on certain pathophysiological aspects of EM, while interferon targets the protopathy; however, PCEA is able to produce long-term effects after one treatment cycle, while interferon requires persistent use and may result in certain side effects. Identification of the optimal modality for the treatment of EM secondary to PV will require further studies.

\section{References}

1. Mitchell SW: On a rare vaso-motor neurosis of the extremities and on the maladies with which it may be confounded. Am J Med Sci 76: 17-36, 1878 . 
2. Alhadad A, Wollmer P, Svensson A and Eriksson KF: Erythromelalgia: Incidence and clinical experience in a single centre in Sweden. Vasa 41: 43-48, 2012.

3. Cohen JS: Erythromelalgia: New theories and new therapies. J Am Acad Dermatol 43: 841-847, 2000.

4. Stricker LJ and Green CR. Resolution of refractory symptoms of secondary erythermalgia with intermittent epidural bupivacaine. Reg Anesth Pain Med 26: 488-490, 2001.

5. Macres S and Richeimer S: Successful treatment of erythromelalgia with intrathecal hydromorphone and clonidine. Clin J Pain 16: 310-313, 2000.

6. Seishima M, Kanoh H, Izumi T, Niwa M, Matsuzaki Y, Takasu A, Ban M and Kitajima Y: A refractory case of secondary erythermalgia successfully treated with lumbar sympathetic ganglion block. Br J Dermatol 143: 868-872, 2000.

7. Michiels JJ, Berneman Z, Schroyens W, Koudstaal PJ, Lindemans J, Neumann HA and van Vliet HH: Platelet-mediated erythromelalgic, cerebral, ocular and coronary microvascular ischemic and thrombotic manifestations in patients with essential thrombocythemia and polycythemia vera: A distinct aspirin-responsive and coumadin-resistant arterial thrombophilia. Platelets 17: 528-544, 2006.

8. McCormack HM, Horne DJL and Sheather S: Clinical applications of visual analogue scales: A critical review. Psychol Med 18 : 1007-1019, 1988.

9. Heidrich H: Functional vascular diseases: Raynaud's syndrome, acrocyanosis and erythromelalgia. Vasa 39: 33-41, 2010.

10. Tefferi A: Polycythemia vera and essential thrombocythemia: 2012 update on diagnosis, risk stratification, and management. Am J Hematol 87: 285-293, 2012.
11. Hisama FM, Dib-Hajj SD and Waxman SG: SCN9A-related inherited erythromelalgia. In: GeneReviews ${ }^{\circledR}$. Pagon RA, Adam MP, Ardinger $\mathrm{HH}$ et al (eds). University of Washington, Seattle, WA, 1993-2015.

12. Huang BT, Zeng QC, Zhao WH, Li BS and Chen RL: Interferon alpha-2b gains high sustained response therapy for advanced essential thrombocythemia and polycythemia vera with JAK2 V617F positive mutation. Leuk Res 38: 1177-1183, 2014.

13. Mørk C, Asker CL, Salerud EG and Kvernebo K: Microvascular arteriovenous shunting is a probable pathogenetic mechanism in erythromelalgia. J Invest Dermatol 114: 643-646, 2000.

14. Mørk C, Kvernebo K, Asker CL and Salerud EG: Reduced skin capillary density during attacks of erythromelalgia implies arteriovenous shunting as pathogenetic mechanism. J Invest Dermatol 119: 949-953, 2002.

15. Kalgaard OM, Clausen OP, Mellbye OJ, Hovig T and Kvernebo K: Nonspecific capillary proliferation and vasculopathy indicate skin hypoxia in erythromelalgia. Arch Dermatol 147: 309-314, 2011.

16. Michiels JJ, Berneman Z, Schroyens W and van Urk H: Aspirin-responsive painful red, blue, black toe, or finger syndrome in polycythemia vera associated with thrombocythemia. Ann Hematol 82: 153-159, 2003.

17. Davis MD, Weenig RH, Genebriera J, Wendelschafer-Crabb G, Kennedy WR and Sandroni P: Histopathologic findings in primary erythromelalgia are nonspecific: Special studies show a decrease in small nerve fiber density. J Am Acad Dermatol 55: 519-522, 2006. 